\title{
FIBER ORIENTATION MEASUREMENTS IN COMPOSITE MATERIALS
}

\author{
R. BLANC ${ }^{1}$, Ch. GERMAIN ${ }^{1}$, J.P. DA COSTA ${ }^{1}$, P. BAYLOU ${ }^{1}$, M. CATALDI ${ }^{2}$ \\ ${ }^{1}$ Equipe Signal et Image, LAPS - UMR 5131 CNRS - ENSEIRB, ENITAB, Université Bordeaux1 \\ 351 cours de la Libération - 33405 TALENCE cedex - FRANCE \\ ${ }^{2}$ Snecma Propulsion Solide, Snecma Group, Les Cinq Chemins 33185 Le Haillan - France
}

\begin{abstract}
Fiber orientation is essential for the physical properties of composite materials. The theoretical parameters of a given reinforcement are usually known and widely used to predict the behavior of the material. However, manufacturing operations such as weaving or needling can produce deviations or fluctuations of fibers around the expected principal directions. These deviations can cause unexpected behavior of the material and should be taken into account in the quality assessment of the material. In this work, we propose an image processing approach to estimate true principal directions and fiber orientation distribution through image analysis of a single section of the material. The method applies to anisotropic materials with several main fiber directions and with cylindrical fibers bundled in threads. A thread-based labeling algorithm has been developed. It allows reliable estimation of the orientation of fibers and threads and provides directional fiber volume ratios. Our method has been successfully applied to the characterization of carbon reinforcement of composite materials.
\end{abstract}

\section{Keywords}

A. Fibres, A. 3-Dimensional reinforcement, B. Anisotropy, B.Optical microscopy. 


\section{Introduction}

The physical properties of fibrous composites are strongly dependant on the quality of their reinforcement. Depending on the characteristics required by the application (e.g. carbon nozzles for propulsion, high performance brake disks, nautical applications, etc.), reinforcement is carried out by weaving, stacking or needling bundles of fibers. The resulting fibrous structure is then densified with an appropriate matrix.
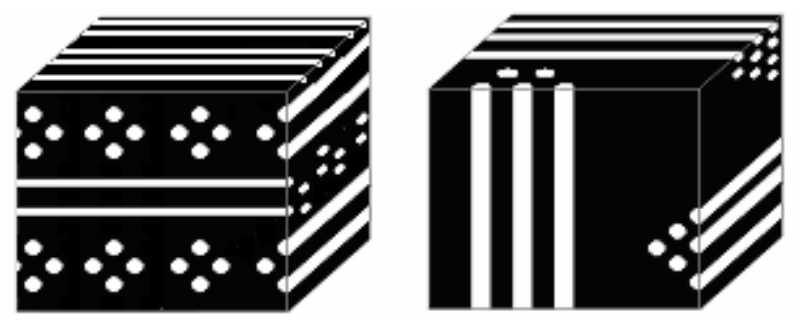

Figure 1 : 3-D representation of material models with 2 and 3 orthogonal directions

Let us consider the general case of a reinforcement made of bundles of fibers - yarns or tows - assembled together along two or three principal directions that are roughly orthogonal one to another (Figure 1). The a priori knowledge of reinforcement architecture can be used to predict the mechanical characteristics of the materials through Finite Elements Models (FEM). Nevertheless, the reliability of this a priori knowledge should be verified since inaccurate FEM inputs may provide biased predictions. Indeed, some steps in the manufacturing process can cause significant deviations and scattering around the expected directions.

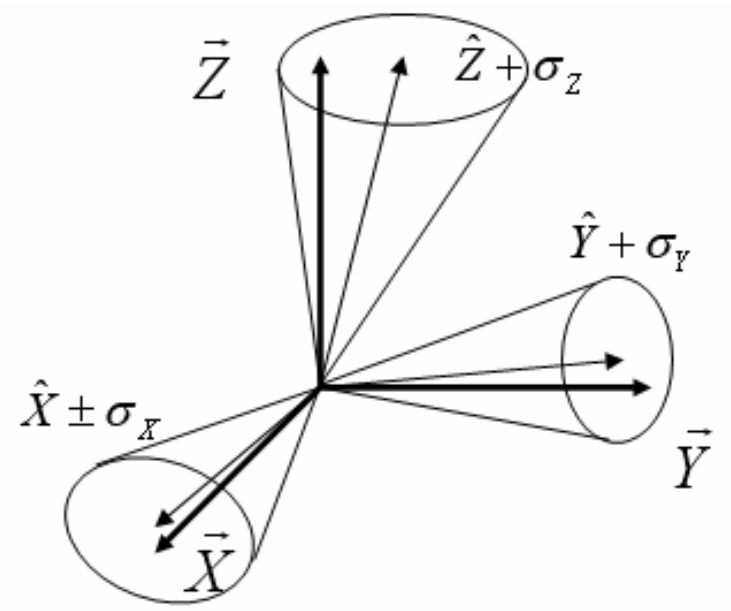

Figure 2: Theoretical principal directions, and measured distributions 
Those deviations originate in fiber undulation caused by weaving, by a twist inside the bundles, by a needling operation, or by some manufacturing defect. Figure 2 illustrates deviations and scattering around desired principal directions for a material showing three orthogonal fiber directions. Knowledge of those deviations would help material designers refine their FEM. It would also allow manufacturers to verify whether the reinforcement is in accordance with predefined standards and finally to improve quality control of production.

Analysis of 2-D or 3-D images of material samples can provide an accurate description of microstructure, particularly of the volume fiber ratio and of fiber orientation.

Obviously, 3-D imaging would be a better solution for estimating such parameters. Micro tomography can produce images with a sufficient resolution [1] to study small diameter fibers - about 5 to 15 micrometers. Unfortunately, this imaging technique is very expensive and can hardly be used for large scale studies.

Another way to obtain 3-D images of the material is the use of confocal microscopy [2][3]. This technique consists in performing multiple optical sections of the sample in order to construct 3-D data. It gives an accurate estimate of the volume fiber ratio and of fiber orientation. Nevertheless, confocal microscopy requires expensive equipment and restrictive sample preparation.

An alternative method, proposed in [4][5], does not require 3-D imaging. It relies on the observation of thin parallel slices. This type of sampling is known in stereology as the dissector [6], and yields worthwhile results. Pattern matching algorithms can establish correspondence of fibers between slices. However, obtaining perfectly parallel slices of an acceptable thickness can prove to be laborious. In this paper, we propose a new image analysis framework based on the processing of a single section, which provides measurements of bundle directions and fiber orientation distribution. We focus on materials showing two or three principal directions roughly orthogonal one to another. We use the a priori knowledge of the expected material structure to compute true principal directions, fiber orientation distribution, and the volume fiber ratio for each principal direction. In the second section we discuss the choice of a section plane allowing 3-D measurements, including the volume fiber fraction. The measurement of the fiber orientation distribution is addressed in the third section. In the fourth section, we exercise our approach to the characterization of a carbon composite material. Finally, in the last section, we conclude and offer some prospects for future work. 


\section{Optimal section plane for material characterization}

\subsection{Measurements on a single section}

Under some conditions related to stereology, the use of a 2-D section for the characterization of a 3-D material is possible. In particular, in order to obtain reliable 3-D measurements, the choice of the section plane is of primary importance. Using a single section, care must be taken that the 2-D measurements truly represents the $3-\mathrm{D}$ parameters.
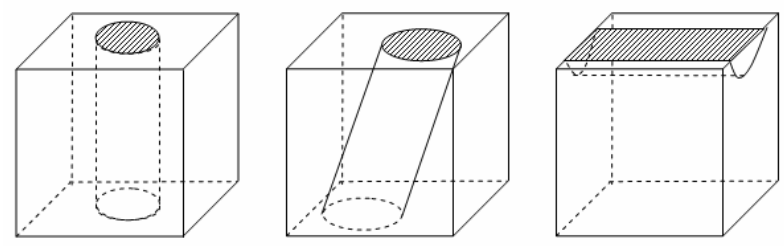

Figure 3: orthogonal, oblique and longitudinal fiber sections

For such a task, the theory of stereology suggests performing an inclined section [7] which avoids cutting fibers lengthwise (see Figure 3). On an inclined section, fibers with circular cross-sections appear as ellipses.

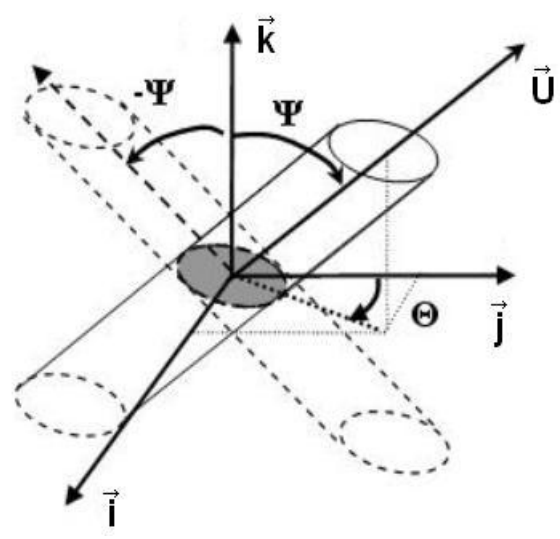

Figure 4: illustration of orientation ambiguity

Ellipse parameters can be used to compute the fiber direction. However, an ambiguity remains. Indeed, as shown in Figure 4, two fibers that are symmetric regarding the section plane remain indistinguishable. Nonetheless, in certain cases, it is possible to overcome this ambiguity on a single section. Mlekusch [8] describes a method for unambiguous fiber orientation measurement in short-fiber-reinforced thermoplastics. In such materials, fibers are arranged so that they are all nearly orthogonal to a specific "normal" direction. To overcome orientation ambiguities, Mlekusch proposes using a section that forms an angle $\alpha$, about $30^{\circ}$, with this normal direction as shown in Figure 5. Indeed, two possible fiber 
orientations can be associated with each elliptic fiber section. With an inclined section plane, only one of these two possible fiber orientations is perpendicular to the normal direction. The ambiguity is thus easily overcome.

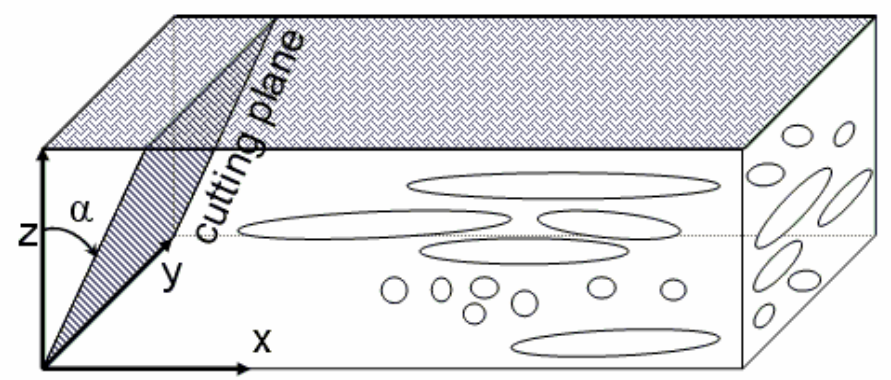

Figure 5: Mlekush oblique section plane

In section 3 we extend this approach to laminates or cloth-based composite materials presenting two or three principal directions.

\subsection{Choice of the section plane}

Recognition and characterization of 3-D shapes on a given 2-D section directly depend on the choice of the section plane. This choice must conform to basic stereological rules [7]:

- no principal direction of the material must be favored,

- the image on the section plane must be representative of the material volume.

The first rule means that the section plane should not be parallel to any principal direction of the material.

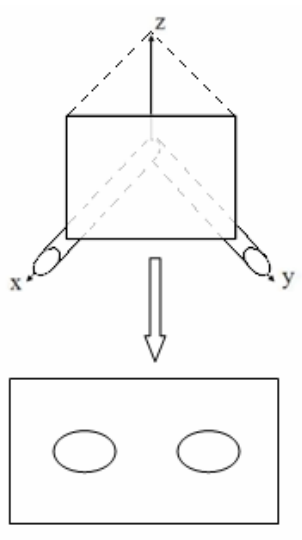

(a)

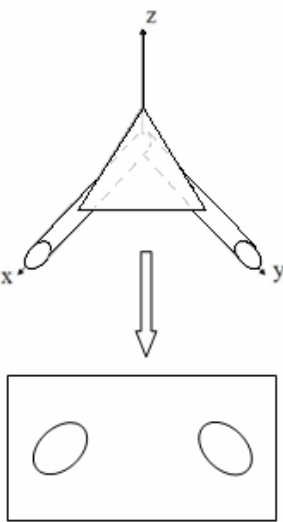

(b)

Figure 6: Illustration of two possible oblique sections and of the corresponding elliptic sections, in the case of two fiber orientations. 
The second implies that the image of the section plane covers a sufficient area to be statistically representative of the different structures within the material. If both requirements are met, 2-D measurements can be used for the estimation of the corresponding 3-D parameters, e.g. the surface and volume fiber fractions.

Among the possible section planes, some cannot be used to correct the fiber direction ambiguity. In the case of materials supposedly presenting two orthogonal directions $x$ and $y$, a section perpendicular to the main plane $(x, y)$ forming a $\pi / 4$ angle with $x$ and $y$ might be an intuitive choice(Figure 6a). However, it would lead to indistinguishable ellipses for both directions. Following the scheme proposed in [8], we choose a section plane which is not perpendicular to the main plane $(x, y)$ (Figure 6b), but which cuts both principal fiber directions with an angle $\Omega$ defined as follows:

$$
\Omega=\cos ^{-1}\left(\frac{1}{\sqrt{3}}\right) \approx 55^{\circ} .
$$

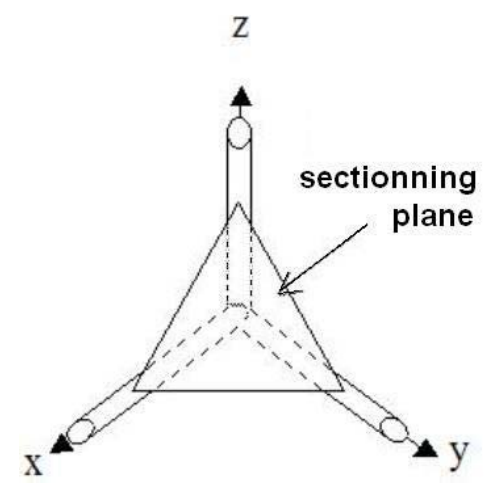

Figure 7: oblique section for three principal fiber directions

For materials showing three orthogonal directions, the same section plane is used. The three main directions are cut with the same angle $\Omega$, as shown in Figure 7 . Hereafter, we will refer to this particular section plane as the 'oblique section plane'. Using such a section ensures that the ellipses from the different principal directions are distinguishable.

For different reinforcement architectures, the general method to determine the oblique section plane is to maximize the differences, i.e. orientations and ellipticities, between ellipses generated by different principal directions. At the same time, care must be taken to avoid longitudinal fiber sections. The oblique section plane also has a number of advantages as concern toward fiber undulation. Indeed, in the case of undulating fibers, an arbitrary section plane is likely to intersect the same fiber several times. Moreover, fiber undulation causes variations in fiber orientation resulting in confusion between fibers from different principal directions. Using the oblique section plane, both risks are reduced. 


\section{Fiber orientation distribution measurement}

Once the section plane is chosen and the sample prepared, reinforcement characterization relies on an image processing algorithm. To remain generally applicable, we address neither sample preparation before image acquisition nor the acquisition technique itself, which are both application dependant. The image processing algorithm is more general. It involves three steps:

- ellipse detection and fiber parameter estimation,

- fiber labeling and thread segmentation,

- computation of characteristics of the reinforcement.

The stages of the approach are summarized in Figure 8.

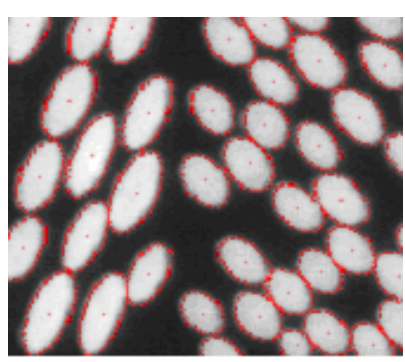

$20 \mu \mathrm{m}$

(a)

Ellipse detection and fiber parameter estimation.

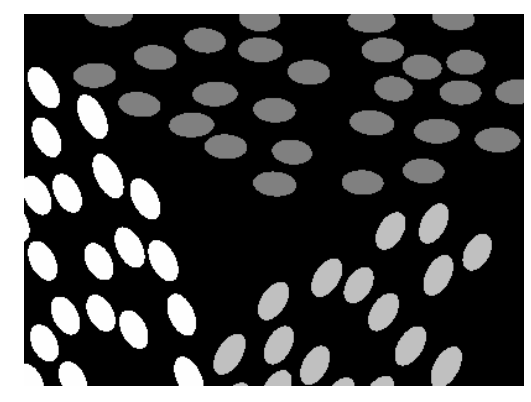

(b)

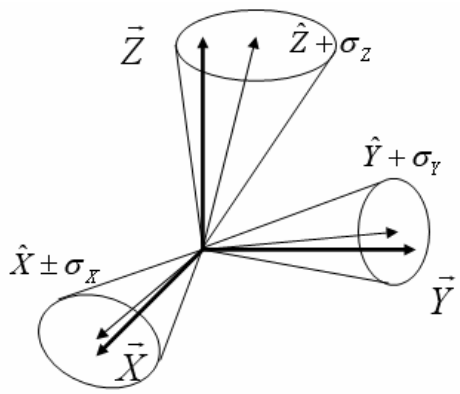

(c)

Fiber labeling and thread segmentation. Computation of the reinforcement (synthetic image, the grey levels stand characteristics. for the various principal directions)

Figure 8: Overview of the approach.

\subsection{Ellipse detection and fiber parameter estimation}

The ellipse detection step depends on the nature and the quality of the section image. Usually, thresholding followed by mathematical morphology operations such as opening or closing [9] allows segmenting most ellipses and splitting those connected. It is assumed here that after sample preparation and image acquisition, the image is segmented so that it shows objects - the elliptic fiber sections - on a background (see Figure 8a).

An appropriate pattern matching algorithm can detect ellipses and extract the collection of ellipse parameters. Many methods exist for ellipse parameter estimation, among them Hough's transform [10] and ellipse fitting [11]. In this paper, the first and second moments of inertia are used. Ellipse axes and 
orientation are computed using the covariance of the ellipse pixel set. Each ellipse is entirely described by its position $(x, y)$ on the section plane, the length of major and minor axes $a$ and $b$ and orientation $\theta$ as shown in Figure 9.

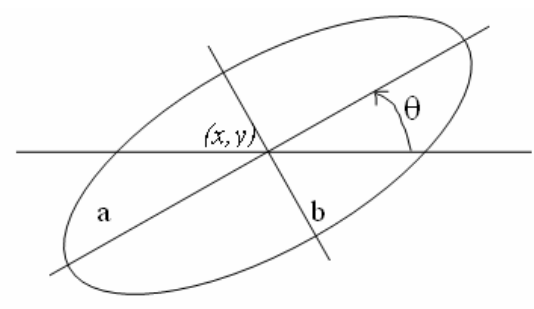

Figure 9: ellipse parameters

$$
\begin{aligned}
& a=\sqrt{2 *\left(a_{11}+a_{22}+\delta\right)} \\
& b=\sqrt{2 *\left(a_{11}+a_{22}-\delta\right)} \\
& \theta=\arctan \left(\frac{2 * a_{21}}{a_{11}-a_{22}+\delta}\right)
\end{aligned}
$$

where: $\delta=\sqrt{\left(a_{11}+a_{22}\right)^{2}-4 *\left(a_{11} a_{22}-a_{12} a_{21}\right)}$

and the $a_{i j}$ are the coefficients of the covariance matrix $A=\left[\begin{array}{ll}a_{11} & a_{12} \\ a_{21} & a_{22}\end{array}\right]$

This method offers a good compromise between complexity and parameter accuracy.

We use 3-D geometry to relate each set of ellipse parameters to the fiber parameters $(\Theta, \Psi)$ defined in Figure 4:

$$
\left\{\begin{array}{c}
\Theta=\theta \\
\cos (\Psi)=\frac{b}{a}
\end{array}\right.
$$

The fiber orientation $\Theta$ is defined in $[0 ; \pi]$. The fiber inclination $\Psi$ belongs to $\left[-\frac{\pi}{2} ; \frac{\pi}{2}\right]$ (see Figure 4) but cannot be evaluated without ambiguity since:

$$
\Psi= \pm \psi^{\prime} \text { where } \psi^{\prime}=\arccos \frac{b}{a} \in\left[0 ; \frac{\pi}{2}\right]
$$

The ambiguity for the fiber direction corresponds to the indeterminacy of the sign of $\Psi$. This ambiguity will be overcome in the following section using the a priori knowledge of material architecture and a fiber labeling algorithm. 


\subsection{Fiber labeling and thread segmentation}

\subsubsection{Principal direction identification: the polar graph}

In order to depict the distribution of fiber orientations, each pair $\left(\theta, \psi^{\prime}\right)$ is mapped on a polar graph.

Each ellipse is located on the polar graph with the argument $2 \theta$ and the modulus $\psi^{\prime} \in\left[0 ; \frac{\pi}{2}\right]$.
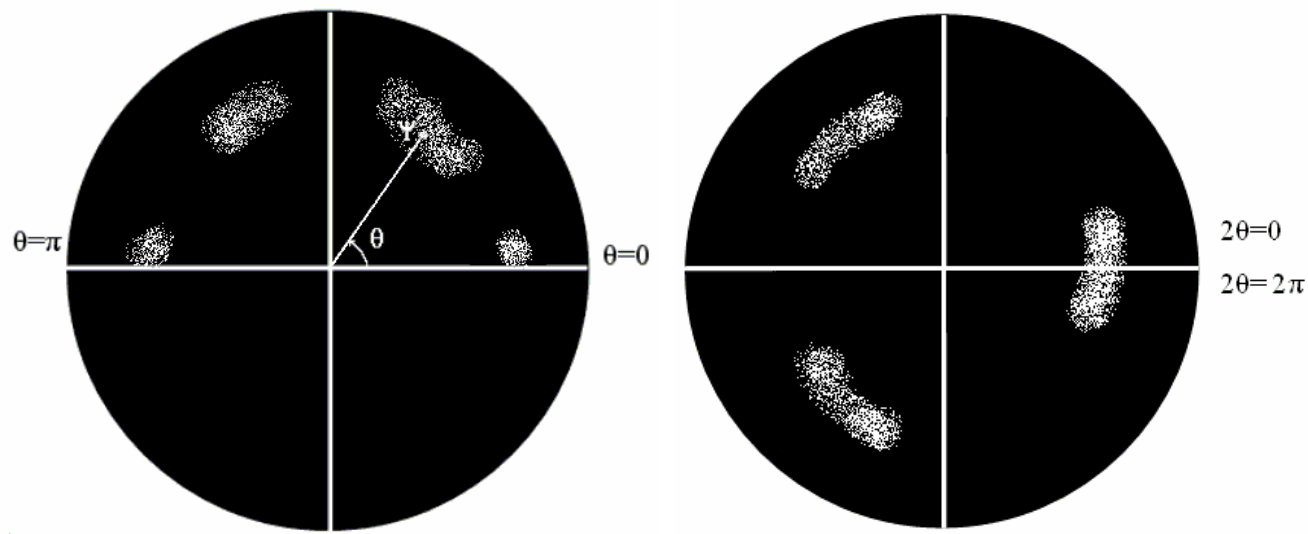

Figure 10: polar graph with simple orientation, and doubled angle

The polar representation of parameter distribution has the advantage of taking the periodic nature of $\theta$ into account. Moreover, taking $2 \theta$ instead of $\theta$ allows avoiding spurious discontinuities (Figure 10). In the case of material presenting multiple directions of anisotropy, the polar graph will show as many blobs as principal directions. Let $N$ be the number of directions. It is necessary to identify each of the $N$ directions i.e. to assign the ellipses of the polar graph to $N$ groups.

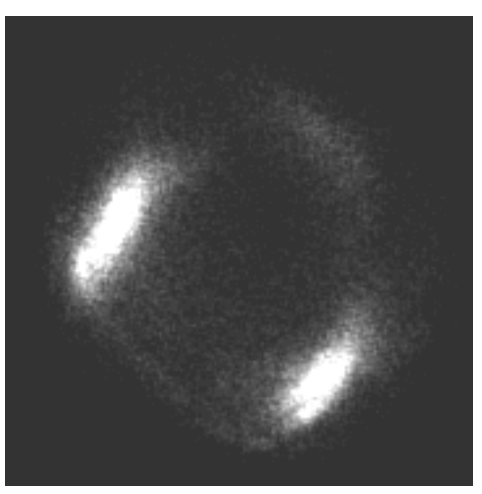

(a)

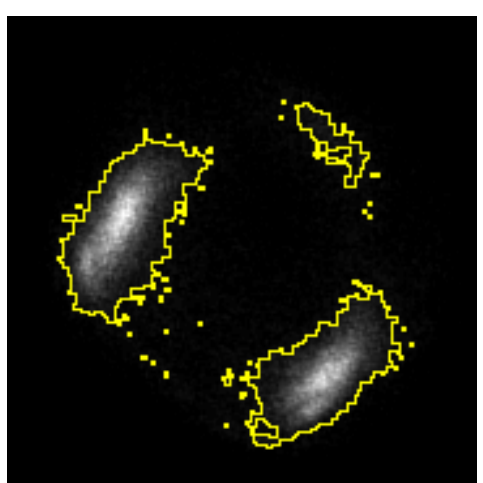

(b)

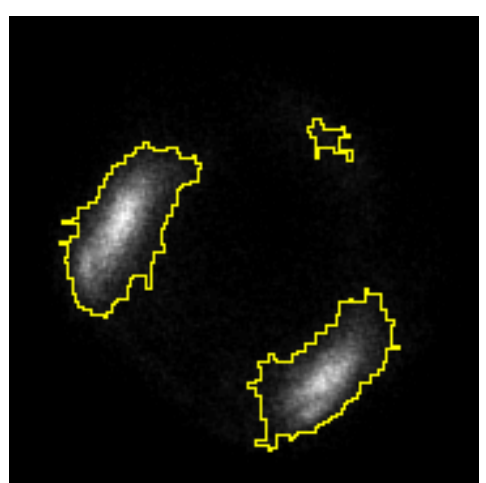

(c)

Figure 11. Processing of the polar graph in the case of $N=3$ principal directions: (a) initial polar distribution, (b) thresholding result, (c) blobs after dilation and filtering. 
For this purpose, we propose converting the polar distribution into a digital image. For each ellipse, the parameters $\left(\theta, \psi^{\prime}\right)$ are converted into integer Cartesian coordinates and the grey level of the corresponding pixel is incremented. The resulting digital image of the polar graph is segmented using a threshold operation. A morphological dilation is then performed on the obtained binary image. Finally, small area blobs are filtered to ensure one and only one blob per principal direction. Figure 11 illustrates these different steps.

The points (i.e. the ellipses) within the limits of a given blob are used to compute the mean direction of this specific blob. Each ellipse of parameters $\left(\theta, \psi^{\prime}\right)$ can be represented by a 3-D vector $\vec{u}$ (Figure 4$)$ :

$$
\vec{u}=\left(\begin{array}{c}
\sin \left(\psi^{\prime}\right) \cos (\theta) \\
\sin \left(\psi^{\prime}\right) \sin (\theta) \\
\cos \left(\psi^{\prime}\right)
\end{array}\right)
$$

The mean direction of a specific blob is given by the vector mean of all vectors inside the blob [12][13]. The mean direction of each blob is still affected by the orientation ambiguity since the mean directions involve $\psi^{\prime}$ instead of $\Psi$ in their expression.

To obtain the correct directions, a priori knowledge on the main directions of the material is required; the relative angles between principal directions and the a priori fiber ratio along each direction are used. Specific steps are applied to determine the principal orientation of the material. Let us note $\vec{U}_{i}, \theta_{i}$ and $\psi_{i}$ the parameters related to the $i^{\text {th }}$ principal direction, $i \in\{1, \ldots, N\}$. For each $i$, the sign of $\psi_{i}$ is not known, thus $\vec{U}_{i}$ can take two different values. The method to determine the correct values for the vectors $\vec{U}_{i}$ involves the following algorithm.

- The first step is to choose arbitrarily the sign of $\psi_{1}$.

- For $i=2$, we determine the correct direction from the two possible values for $\vec{U}_{2}$. The choice of the right value is achieved by comparing the two possible relative angles $\operatorname{Angle}\left(\vec{U}_{1}, \vec{U}_{2}\right)$. The correct direction is given by the relative angle that is the closest to the a priori value.

- $\quad$ The same procedure is applied to all $\vec{U}_{i}, i \in\{3, \ldots, N\}$. At each step, the a priori knowledge of the approximate relative angles ensures the uniqueness of the solution. 
In the case of a woven reinforcement with $\mathrm{N}=2$ or $\mathrm{N}=3$ main directions, this task is straightforward as warp and fill yarns are generally supposed orthogonal.

Concerning the first step, changing the sign of $\psi_{1}$ leads to replacing each vector $\vec{U}_{i}, i \in\{1, \ldots, N\}$ by its symmetric regarding the section plane. This results in the exact symmetric layout, thus in strictly equivalent orientation statistics.

At this step, using the a priori knowledge about the theoretical main directions of the reinforcement, the sign of the angles $\psi_{i}$ and thus the principal directions $\vec{U}_{i}, i \in\{1, \ldots, N\}$, are determined. However, at this point, there is no correspondence yet between each individual fiber and the principal direction to which it belongs.

A solution would be to establish this correspondence on the polar distribution, using for instance a $k$ means type algorithm [14] to classify the fibers in $\mathrm{N}$ groups depending on the nearest principal direction. Unfortunately, many disorientations and tearings usually exist within the material, for example due to the fiber waviness or to some needling operation. This disorder may cause an overlap of the blobs in the polar graph and lead to misclassifying some fibers.

In order to avoid this situation, the spatial layout of fibers is taken into account. More precisely, the notion of threads is introduced. Is is assumed that all fibers of a given thread are oriented in the same main direction. Therefore, a thread segmentation algorithm was developed to establish the correspondence between fibers and their true main direction.

Note:

The polar graph can also be used to estimate the quality of the oblique section plane. Indeed, if the section plane does not favor any of the $N$ material principal directions, the polar graph will show $N$ well defined blobs. On the contrary, if the section plane is far from the oblique section plane, blobs merge into one single continuous area.

\subsubsection{Thread segmentation}

In order to segment the image into threads, a fiber labeling process is introduced. This labeling process associates each fiber with its thread and thereby with its corresponding main direction. Hence, labeling a fiber also cancels the directional ambiguity related to its particular orientation.

The fiber labeling process consists in three steps: the thread kernel selection, the propagation within the thread and the processing of the remaining fibers. 


\section{Thread kernel selection}

This step consists in selecting the fibers that show a direction close to one of the mean vectors $\vec{U}_{i}$, i.e. the fibers which are nearly parallel to one of the true principal directions. The selection is done according to a specified threshold angular value defining a cone around each main direction. The fibers with orientations lying inside the cone constitute the fiber kernel of the corresponding region of the polar graph. Selection must be very strict in order to avoid choosing fibers within the overlapping zone of two regions of the polar graph.

As the problem of direction ambiguity in each region was solved at the previous stage, the correct direction, i.e. the sign of the parameter $\Psi$, can be assigned to each fiber in these kernels.

However, to avoid labeling isolated fibers during this first step, only those kernel fibers surrounded by at least six valid neighboring fibers are labeled. The number six corresponds to the number of direct neighbors in a perfect arrangement of cylinders with a maximum of compactness.

The criteria for the validation of neighboring fibers are the following:

- an angle: two fibers are neighbors if their directions are similar. Similarity is established based on a threshold angle.

- a distance: fiber $\mathrm{A}$ is a neighbor of fiber $\mathrm{B}$ if the distance between the centre of ellipse $\mathrm{A}$ and the axis of fiber B are under a pre-defined threshold.

In practice, at this stage, less than $1 \%$ of the fibers constitute the thread kernels. Experimental tests have shown that kernel fibers usually lie in the center of threads and that each thread contains at least one kernel fiber.

\section{Propagation within a thread}

For this second step, the fact that fibers are bundled into threads is taken into account. The mean direction of a thread, as it contains hundreds of fibers, is close to one of the main directions of the material.

Moreover, all the fibers in a thread necessarily belong to the same principal direction.

The propagation process consists in spreading iteratively the labels of the kernel fibers to the neighboring fibers, using the rules defined above (i.e. angle and distance criteria) but with less selective thresholds. Thus, bundles of labeled fibers grow around the kernels. When two fiber bundles join each other, their mean directions are compared to ascertain if they belong to the same main direction. If these directions are similar, the two bundles are merged.

\section{$\underline{\text { Residual fibers }}$}


At the end of the propagation process, some fibers may not be labeled. Those remaining fibers are generally located outside the threads, and far from the main directions of the polar graph. As they usually are broken fibers, it is possible to reject them or apply a final step.

This final step consists in assigning them a label considering their directions and the mean orientation of the closest labeled thread.

\subsection{Fiber and thread characterization}

At this step, the true 3-D orientation of each fiber is unambiguous and a fiber database exists from which various features can be computed at various scales. Here follows a non exhaustive list of such features.

- Statistics on principal directions:

o true relative angles between the principal directions,

o scattering of the fiber directions for each principal direction,

o volume fraction of fibers for each principal direction.

- $\quad$ Statistics at thread scale:

o mean and variance of the number of fibers within each thread,

o scattering of fiber directions between or within the threads, supplying information about the fiber undulation or the twist within the yarns.

- $\quad$ Statistics at fiber scale:

o mean and variance of the fiber diameter,

o fiber volume fraction of the material.

\section{Application to carbon fiber composite material}

The approach developed in this paper was applied to images of carbon composite materials before densification. Carbon fibers have a circular cross-section with a theoretical mean diameter of $7 \mu \mathrm{m}$.

Fibrous structure is made of threads containing a thousand fibers.

The material used here is a needled $\left[0^{\circ} / 90^{\circ}\right]$ laminate. Layers are composed of parallel threads in $\mathrm{X}$ and $\mathrm{Y}$ directions alternatively, $\mathrm{X}$ and $\mathrm{Y}$ being orthogonal. The layers are needled in a third orthogonal direction $\mathrm{Z}$. Bundles in the $\mathrm{Z}$ direction, as they are created by the needling operation, contain fewer fibers and are less organized. 
As the fiber structure of this material shows three orthogonal principal directions, images were acquired following an oblique section plane as explained in Figure 7.

An optical microscope at a resolution of $0.5 \mu \mathrm{m}$ per pixel was used. Each image contains $512 * 512$ pixels. A large field of $20 * 30$ images was acquired using an automatic process, for a total surface of roughly $40 \mathrm{~mm}^{2}$ and $10^{5}$ fibers. Figure 12 shows a sample of such an image field. Fibers are grey; the layered structure of the reinforcement appears. Several threads of different directions can be identified before processing as clusters of ellipses.
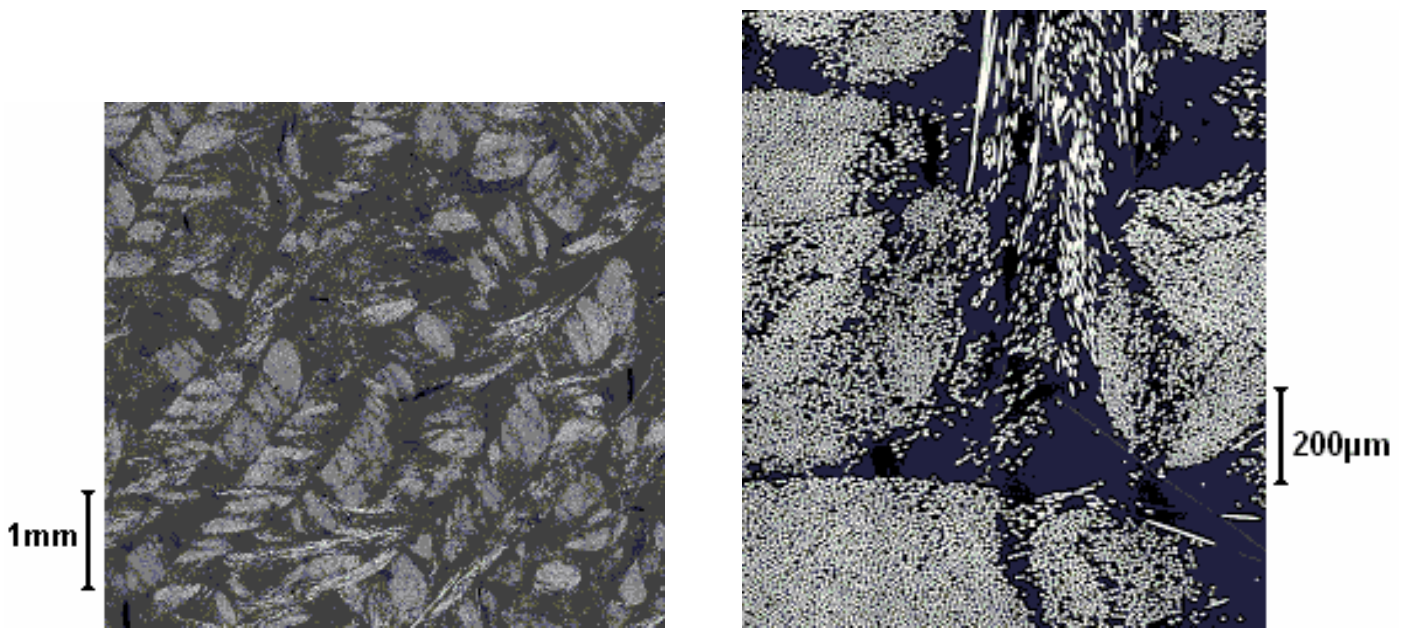

Figure 12: images of the material at various scales.

\subsection{Ellipse detection}

Figure $8 \mathrm{a}$ shows the result of fiber segmentation. The red edge indicates the boundary of each ellipse.

This figure shows two different threads with different directions. The mean fiber diameter measures

$7.1 \mu \mathrm{m}$ with a standard deviation of $0.7 \mu \mathrm{m}$. The volume fiber fraction is estimated at $20.3 \%$.

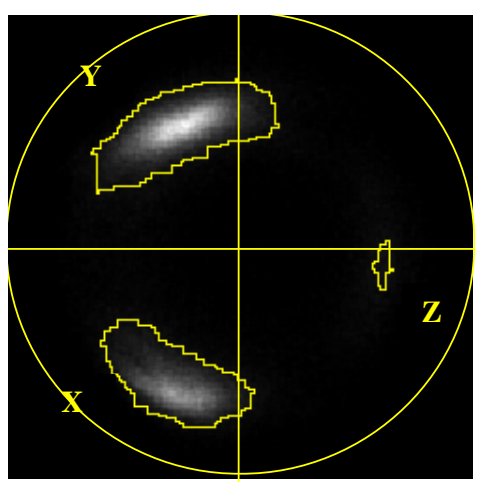

Figure 13: Polar graph after main direction identification 


\subsection{Fiber labeling and thread segmentation}

The ellipse parameters, obtained for each fiber, are plotted on the polar graph Figure 13. The three main

Fig.13 directions $\mathrm{X}, \mathrm{Y}$ and $\mathrm{Z}$ of the material appear on this graph as lighter regions.

Figure 13 shows, in yellow outlines, the result of the segmentation of the main directions on the polar graph. Their mean orientations $\vec{X}, \vec{Y}$ and $\vec{Z}$ were first computed. Then, the main direction identification and the related ambiguity correction were carried out, considering that the three directions are theoretically orthogonal, and that the $\mathrm{Z}$ direction contains fewer fibers.

The following estimates for the relative angles between the main directions were obtained:

$$
\operatorname{Angle}(\vec{X}, \vec{Y})=87.8^{\circ} ; \operatorname{Angle}(\vec{X}, \vec{Z})=81.9^{\circ} ; \operatorname{Angle}(\vec{Y}, \vec{Z})=90.2^{\circ} \text {. }
$$

The estimations of the principal directions confirmed that the two directions of the weave were nearly orthogonal, but the mean orientation of the $\mathrm{Z}$ bundels shifted slightly compared to the expected orthogonal result. This can be explained by the fact that the needling operation is performed while the stack of weaves is moving. This movement has a significant impact on the Z-fiber mean direction.

For the thread segmentation algorithm, fibers whose relative angle with a principal direction is below $15^{\circ}$ were selected. Moreover, such fibers had to have at least 6 neighbors. The neighboring parameters were set at $10^{\circ}$ for the angle threshold and $\sqrt{2}$. fiber_ radius for the distance threshold. Experiments showed that in using such parameter values, at least one kernel fiber is present in each thread and even in each $\mathrm{Z}$ bundle. At this stage, only $0.2 \%$ of the fibers were labeled. This low percentage ensured the relevance of the kernel.

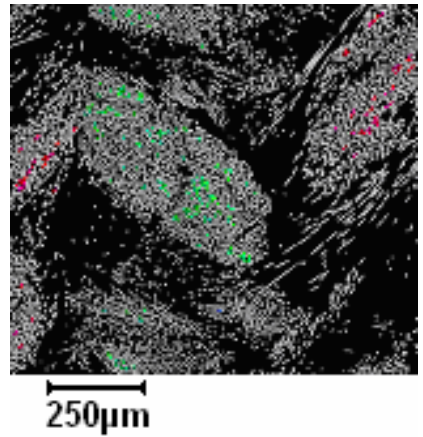

(a)

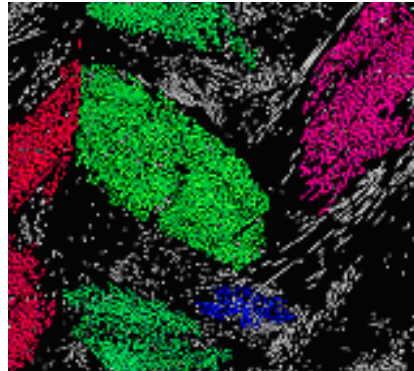

$250 \mu \mathrm{m}$

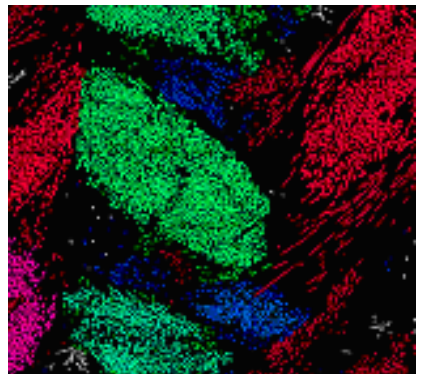

$250 \mu \mathrm{m}$

(b)

(c)

Figure 14: intermediate results of the thread segmentation algorithm: (a) kernel selection, (b) thread segmentation, (c) processing of the remaining fibers 
Then, from these kernel fibers, the propagation step assigned a label to $85 \%$ of the fibers. The different steps of the thread segmentation are illustrated in Figure 14.

Remaining fibers were processed considering their directions and the orientation of the closest labeled thread. The $\mathrm{Z}$ direction was favored using higher threshold parameters. That allowed highlighting the specificity of the needling process. Indeed, as the needle draws $\mathrm{X}$ or $\mathrm{Y}$ fibers in the $\mathrm{Z}$ direction, orientations of Z-fibers are much more scattered than are the directions of the weaves. Figure 15 shows the final result of the labeling process. X-fibers appear in red, Y-fibers in green, and Z-fibers in blue.

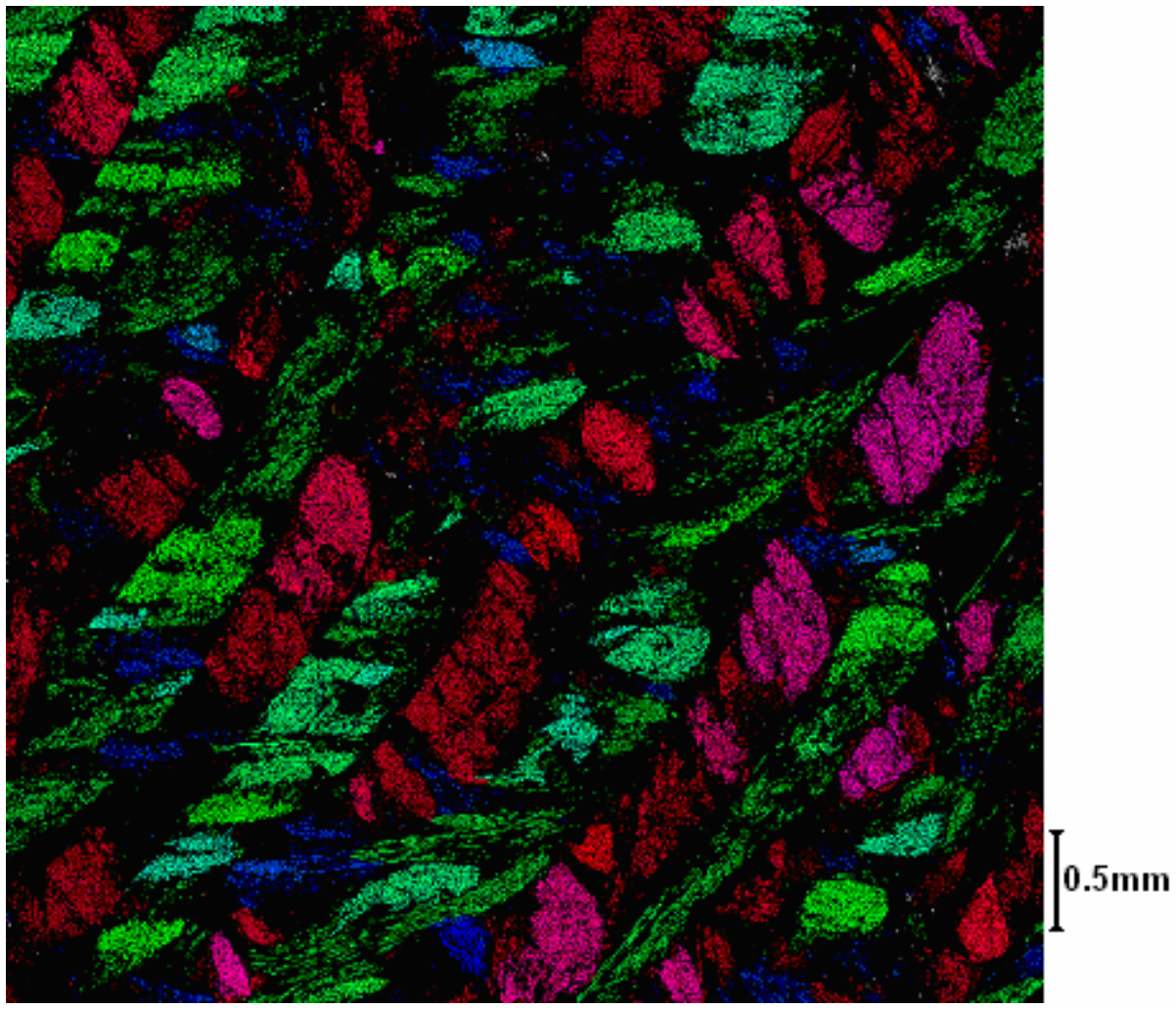

Figure 15: Image of the labeled material

This kind of representation helps visualizing the reinforcement and verifying whether it is consistent with its expected structure or not.

\subsection{Thread and fiber characterization}

The measured mean diameter, which is $7.1 \mu \mathrm{m}$ with standard deviation of $0.7 \mu \mathrm{m}$, is consistent with carbon fiber production standards.

The global fiber ratio of $20.3 \%$ was confirmed by density measures. This global ratio was broken down into directional ratios: $9.1 \%$ for the $\mathrm{X}$ direction, $9.5 \%$ for $\mathrm{Y}$ and $1.7 \%$ for $\mathrm{Z}$. These directional ratios reflected the fact that the two weave directions were equally represented. 
The measured relative angles, Angle $(\vec{X}, \vec{Y})=87.8^{\circ}, \operatorname{Angle}(\vec{X}, \vec{Z})=81.9^{\circ}, \operatorname{Angle}(\vec{Y}, \vec{Z})=90.2^{\circ}$, between the principal directions were also found to be in accordance with the desired design of the reinforcement.

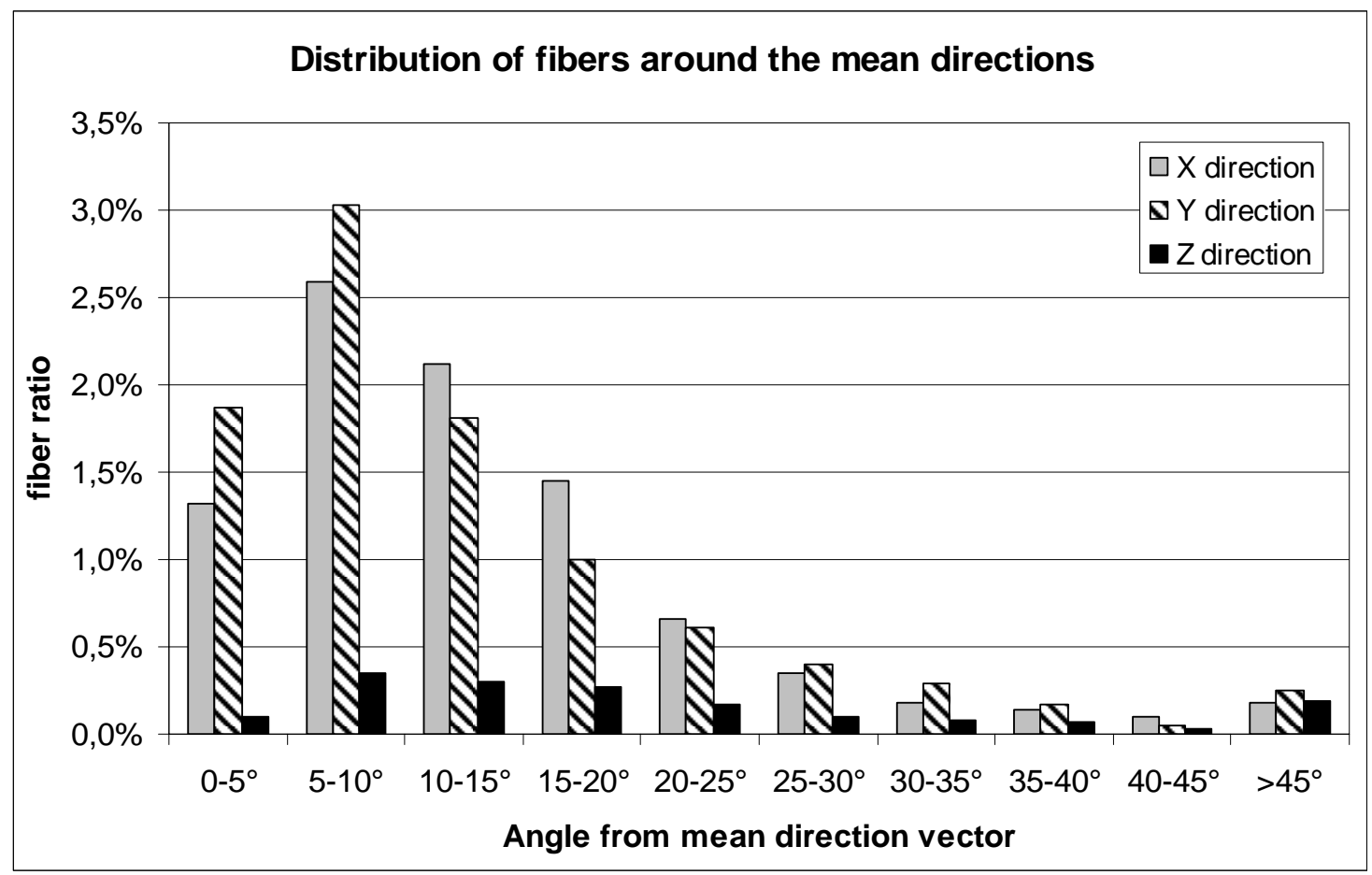

Figure 16: statistics on fiber orientation distribution

The statistics on orientation dispersion around the main directions provide information about fiber waviness. Figure 16 shows the histogram of fiber orientation distribution around each principal direction. It appears that fiber orientations are significantly scattered around the principal directions.

\section{Conclusion}

In this paper, we proposed an image analysis framework for the characterization of fiber composite reinforcements. This framework is based on the observation of a single section of the material and mainly aims at measuring the effective principal directions of the reinforcement, the volume fiber ratio in each direction and the fiber orientation distribution. Three stages of the framework were detailed.

First, the influence of the section plane on the reliable estimation of volume parameters using 2-D data was discussed. Instructions for choosing the section plane were given. Then, a polar representation of fiber parameter distribution was used in order to identify principal material directions. Finally, a thread segmentation algorithm was presented which results in the complete classification of the fibers into the principal directions of anisotropy in the material. This segmentation provides the distribution of fiber 
orientations. The full segmentation of fibers also provides a large set of geometrical characteristics of the reinforcement.

The approach was successfully applied to the characterization of specific carbon/carbon composite materials. It is currently used in an industrial application for the quality assessment of carbon reinforcement for composite materials.

Other aspects related to material characterization are currently under study. One of them concerns the quantification of the reliability of 3-D parameter estimation by image processing of 2-D sections. In other words, the statistical influence of the 2-D section on the variance of the estimation of any material 3-D parameter is being evaluated. The links between the surface of acquisition and the estimation variance are under study as well. Other prospective works concern the generalization of such statistical studies to different kinds of materials e.g. metallic materials, and materials showing different types of structures e.g. blob-like, fibrous or cubic structures.

\section{Acknowledgements}

This work has been partly funded by the Region Aquitaine, with the financial and technical support of the SNECMA group. The authors also wish to thank Lee Valente for her valuable help in writing this paper.

\section{References}

1. Coindreau O, Vignoles G, Cloetens. Direct 3D microscale imaging of carbon-carbon composites with computed holotomography. Nucl. Instr. And Meth. in Phys. Res. B 200, 2003:308-314.

2. Eberhardt C, Clarke A. Fiber-orientation measurements in short-glass-fiber composites. Part I: automated, high-angular-resolution measurement by confocal microscopy. Composites Science and Technology, 2001; 61-1389.

3. Lee KS, Lee SW, Youn JR, Kang TJ, Chung K. Confocal Microscopy Measurement of the Fiber Orientation in Short Fiber Reinforced Plastics. Fibers and Polymers, 2001; 2(1): 41-50.

4. Lee YH, Lee SW, Youn JR, Chung K, Kang TJ. Characterization of fiber orientation in short fiber reinforced composites with an image processing technique. Mat Res Innovat 2002; (6):65-72

5. Davidson NC, Clarke AR, Archenhold G. Large-area, high-resolution image analysis of composite materials, Journal of Microscopy, 1997; 185(2):233-242.

6. Sterio DC, The unbiased estimation of number and sizes of arbitrary particles using the dissector, Journal of Microscopy, 134 Pt 2:127-36, May 1984. 
7. Russ JC, Dehoff RT, Practical Stereology 2nd edition, Plenum Press, New York, NY, Nov. 2000.

8. Mlekusch B. Fiber orientation in short-fiber-reinforced thermoplastics II. Quantitative measurements by image analysis. Composites Science and Technology. 1999; 59-547.

9. Russ JC. The image processing handbook, 3rd ed., CRC Press, Boca Raton, FL,1998.

10. McLaughlin RA, Randomized Hough Transform: Improved ellipse detection with comparison, Pattern Recognition Letters, 1998; (19):299-305.

11. Ahn SJ, Rauh W, Warnecke HJ, Least-square orthogonal distances fitting of circle, sphere, ellipse, hyperbola, and parabola, Pattern Recognition, 2001; (34): 2283-2303.

12. Mardia KV, Statistics of Directional Data, Academic Press, London, New York, 1972

13. Germain C, Da Costa JP, Lavialle O Baylou P. Multiscale estimation of vector field anisotropy.

Application to texture characterization, Signal Processing, 2003; (83): 1487-1503.

14. Diday E., Simon J.C., Clustering Analysis, Chapter Digital Pattern Recognition, K.S. Fu Ed., Springer Berlin, 1976. 